This item was submitted to Loughborough's Research Repository by the author.

Items in Figshare are protected by copyright, with all rights reserved, unless otherwise indicated.

\title{
Opportunities and constraints of presently used thermal manikins for thermo- physiological simulation of the human body
}

\section{PLEASE CITE THE PUBLISHED VERSION}

http://dx.doi.org/10.1007/s00484-015-1041-7

\section{PUBLISHER}

(C) Springer

VERSION

AM (Accepted Manuscript)

\section{PUBLISHER STATEMENT}

This work is made available according to the conditions of the Creative Commons Attribution-NonCommercialNoDerivatives 4.0 International (CC BY-NC-ND 4.0) licence. Full details of this licence are available at: https://creativecommons.org/licenses/by-nc-nd/4.0/

\section{LICENCE}

CC BY-NC-ND 4.0

\section{REPOSITORY RECORD}

Psikuta, Agnes, Kalev Kuklane, Anna Bogdan, George Havenith, Simon Annaheim, and Rene M. Rossi. 2019. "Opportunities and Constraints of Presently Used Thermal Manikins for Thermo-physiological Simulation of the Human Body". figshare. https://hdl.handle.net/2134/18617. 


\section{Opportunities and constraints of presently used thermal manikins for thermo-physiological simulation of the}

human body

Agnes Psikuta ${ }^{1}$,Kalev Kuklane ${ }^{2}$, Anna Bogdan ${ }^{3,4}$, George Havenith ${ }^{5}$, Simon Annaheim ${ }^{1}$, René M. Rossi ${ }^{1}$

1) Empa, Laboratory for Protection and Physiology,Lerchenfeldstrasse 5, 9014 St. Gallen, Switzerland

2) Ergonomics and Aerosol Technology, Design Sciences, Lund University, PO Box 118, 22100 Lund, Sweden

3) Thermal Load Laboratory, Central Institute for Labour Protection-PIB, Czerniakowska 16, 00-701 Warsaw, Poland

4) Air Conditioning and Heating Department, Faculty of Environmental Engineering, Warsaw University of Technology, Nowowiejska 20, 00-653 Warsaw, Poland

5) Environmental Ergonomics Research Centre, Loughborough Design School, Loughborough University, Loughborough LE11 3TU, UK

Corresponding author:

Email: agnes.psikuta@empa.ch

Phone: +41 587657673

Fax: +41587656962

\section{ABSTRACT}

Combining the strengths of an advanced mathematical model of human physiology and a thermal manikin is a new paradigm for simulating thermal behaviour of humans. However, the forerunners of such adaptive manikins showed some substantial limitations. This project aimed to determine the opportunities and constraints of the existing thermal manikins when dynamically controlled by a mathematical model of human thermal physiology.

Four thermal manikins were selected and evaluated for their heat flux measurement uncertainty including lateral heat flows between manikin body parts and the response of each sector to the frequent change of the set-point temperature typical when using a physiological model for control.

In general, all evaluated manikins are suitable for coupling with a physiological model with some recommendations for further improvement of manikin dynamic performance. The proposed methodology is useful to improve the performance of the adaptive manikins and help to provide a reliable and versatile tool for the broad research and development in domains of clothing, automotive and building engineering.

Keywords: thermal manikin, adaptive manikin, human simulator, physiological simulation 


\section{INTRODUCTION}

Thermal manikins are the most realistic devices for heat and mass transfer measurements in clothing due to their anatomic shape and their ability to sweat and move. These versatile evaluation instruments are nowadays implemented in a wide range of disciplines including clothing research and manufacturing, the automobile industry, and the environmental engineering of artificial microclimates for workplaces and homes.

Presently, manikins are usually operated at uniform steady-state surface temperatures and homogenous sweat rates in comparative measurements, for example according to standards, such as ASTM F 1291-05:2005 (ASTMF129105 2005), ASTM F 1868-02:2005 (ASTMF2370-05 2005), ISO15831:2004 (ISO15831 2004) and ISO9920:2007 (ISO9920 2007). Nevertheless, various attempts have been undertaken to mimic the thermal response of a human more realistically, for example, by setting uniform heat fluxes to simulate different workloads (Gao et al. 2012; Keiser et al. 2008), or non-uniform surface temperatures over the body, such as cooler hands and feet (McCullough 2002; McCullough et al. 1985), or uniform surface temperature change over time (Tanabe et al. 1994). These attempts indicate the growing interest in using manikins to adequately simulate the effect of clothing and environmental exposures on human thermal responses such as body core temperature and skin temperature distribution, onset of vasomotor reactions, sweating and shivering.

Combining the strengths of an advanced mathematical model of human physiology and a thermal manikin is a new paradigm for simulating thermal behaviour of humans. Ideally, a new-generation adaptive manikin should 'sense' and respond dynamically to the thermal environment as humans do. Forerunners of such systems have already been developed for evaluation of the comfort in vehicles (Farrington et al. 2004), for testing clothing and sleeping systems (Blood and Burke 2010; Burke et al. 2009; Psikuta et al. 2008; Psikuta et al. 2013; Redortier and Voelcker 2010) and for assessment of indoor microclimates (Foda and Siren 2012a; Foda and Siren 2012b; Nilsson 2004). All these attempts used various physical thermal devices and physiological models, and they were validated by comparison to the human thermal response obtained in dedicated human trials. Validation of an adaptive manikin is a challenging process, which requires not only the understanding of the human trial protocol and measurement methods (e.g. calibration and ambient temperature influencing skin temperature and heat flux measurement, clothing fit and body movement as influential factors on heat and mass transfer at human skin) (Niedermann et al. 2014; Psikuta et al. 2012; Psikuta et al. 2014) but also the performance and limitations of both coupled elements, i.e. the thermal manikin and the physiological model, separately. The adaptive manikin studies to date showed only a very limited number of validation cases, in a narrow range of conditions and outlined some disagreements between the human experimental data, the physiological model alone and the adaptive manikin results. However, none of the studies addressed the source of potential discrepancies and limitations of an adaptive manikin in relation to the thermal manikin reliability and performance.

This project aimed to determine the opportunities and constraints of the existing thermal manikins with regards to their functionality when controlled by a mathematical model of human thermal physiology. The thermal characteristic of each manikin included in the study was determined using the same measurement setup, experimenter and method for consistency. The measurement protocols addressed the specific aspects of manikin 
performance when controlled dynamically. These aspects included the method and the measurement uncertainty of the heat flux released from the sectors of the manikin including lateral heat flows between manikin body parts. Secondly, the response of each sector and its dedicated control system to the frequent change of the set-point temperature, which is a consequence of a physiologically floating skin temperature, was characterized. Specifically, the reaction during heating and cooling and the dynamic response to the step changes of the surface temperatures that are typical when using a physiological model for control were investigated.

\section{METHODS}

\section{Operating principle of thermo-physiological human simulators}

A thermo-physiological human simulator comprises of a thermal manikin that is controlled by a model of human thermal physiology using a feedback loop. In this way, the manikin is programmed to respond to the thermal environment as an average human being (average body size, body composition and fitness, not acclimatized). The coupling method of the manikin and the model is based on real-time iterative exchange of the relevant data between the manikin and the model. On one hand, the skin temperatures and sweat rates from the physiological model are used to control the manikin. On the other hand, the heat fluxes measured by the manikin are used as a feedback representing the amount of heat exchanged with the environment in the present climate and clothing conditions (Psikuta et al. 2008). Another coupling strategy is to provide the metabolic heat production (set as a heat flux in the manikin system) and sweat rate for each body part and the resultant manikin surface temperature is the feedback parameter to the physiological model (Curran et al.). This method, however, may be prone to inaccurate estimations of metabolic heat production over the body parts dependent on the activity type and intensity, which heavily impacts results. Other physiological and perceptual parameters derived from the physiological model, such as core temperature, skin blood flow, heart rate and thermal sensation, are also available in both coupling methods (Psikuta 2009). To be able to successfully couple the full body manikins with a physiological model, the manikin precision and accuracy under transient conditions, such as temporally and spatially varying surface temperature, heat loss and sweating, must be ensured. The physiological model by Fiala was used in this study to simulate typical and extreme physiological parameters as a reference for manikin thermal evaluation (Fiala et al. 2012; Psikuta et al. 2012). This model underwent an extensive validation process at the development and use stages, confirming its accuracy and precision (Psikuta et al. 2012).

\section{Manikins}

Four types of thermal manikins were investigated in this study (Figure 1 and Table 1). 
Table 1. Thermal manikins investigated in this study.

\begin{tabular}{|c|c|c|c|c|c|}
\hline Manikin & $\begin{array}{c}\text { Manufacturing } \\
\text { year }\end{array}$ & Laboratory & $\begin{array}{c}\text { Number of } \\
\text { sectors }\end{array}$ & $\begin{array}{c}\text { Max. power } \\
\text { output }\end{array}$ & Reference \\
\hline $\begin{array}{l}\text { Diana (PT } \\
\text { Teknik, } \\
\text { Denmark) }\end{array}$ & 1992 & $\begin{array}{l}\text { Central Institute } \\
\text { for Labour } \\
\text { Protection in } \\
\text { Poland }\end{array}$ & 16 sectors & $200 \mathrm{~W} / \mathrm{m}^{2}$ & $\begin{array}{c}\text { (Konarska et al. } \\
\text { 2007) }\end{array}$ \\
\hline Tore & 1980 & $\begin{array}{l}\text { Lund University in } \\
\text { Sweden }\end{array}$ & 17 sectors & $350 \mathrm{~W} / \mathrm{m}^{2}$ & $\begin{array}{c}\text { (Kuklane et al. } \\
\text { 2006) }\end{array}$ \\
\hline $\begin{array}{l}\text { Newton } \\
\text { (MTNW, USA) }\end{array}$ & 2003 & $\begin{array}{l}\text { Loughborough } \\
\text { University in UK }\end{array}$ & $\begin{array}{c}32 \text { sectors and } 2 \\
\text { guards (upper } \\
\text { thighs) }\end{array}$ & $800 \mathrm{~W} / \mathrm{m}^{2}$ & $\begin{array}{l}\text { (Havenith et al. } \\
\text { 2008) (Havenith } \\
\text { et al. 2013) }\end{array}$ \\
\hline $\begin{array}{l}\text { Sweating Agile } \\
\text { thermal Manikin } \\
\text { SAM }\end{array}$ & 2001 & $\begin{array}{l}\text { Empa in } \\
\text { Switzerland }\end{array}$ & $\begin{array}{l}22 \text { sectors and } 9 \\
\text { guards (face, } \\
\text { elbows, hands, } \\
\text { knees, feet) }\end{array}$ & $600 \mathrm{~W} / \mathrm{m}^{2}$ & $\begin{array}{l}\text { (Richards and } \\
\text { McCullough } \\
\text { 2005) (Psikuta } \\
\text { 2009) }\end{array}$ \\
\hline
\end{tabular}

In all manikins, the manikin surface temperature is measured by resistance wires embedded in the outermost protective coating (Diana, Newton, SAM) or secured by a protective thin tape (Tore). The heating wires or foils are located on the inner side of the manikin shells apart from manikin Diana in which the same resistance wire is used to heat up and measure its surface temperature in alternate cycles. All manikins were temperature calibrated according to standard protocols used individually in each laboratory. Beside SAM, none of the manikins was provided with an active sweating system. This fact constrains their use for warm environments and higher metabolic rates; a sweating function would be necessary when coupled with a physiological model. 


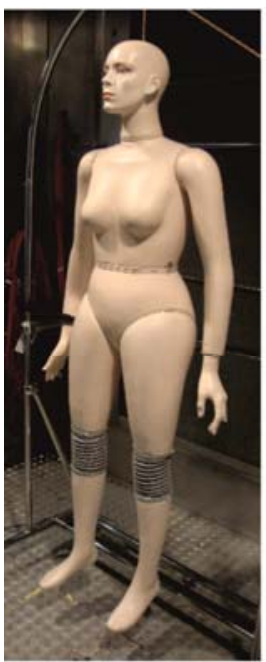

a) Diana

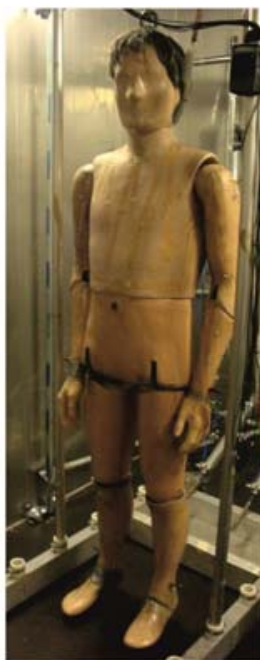

b) Tore

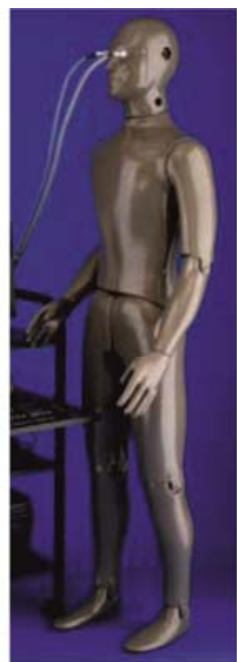

c) Newton

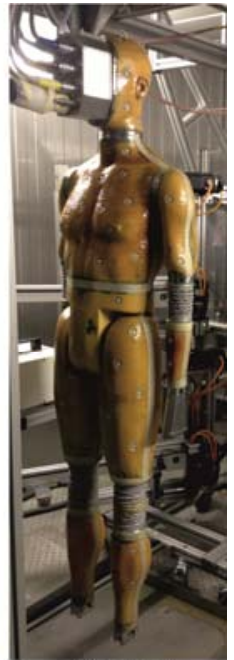

d) SAM

Figure 1. Four manikins used in the study, namely, Diana (Peter Trans, Denmark) from Central Institute for Labour Protection in Poland (a), Tore from Lund University in Sweden (b), Newton (MTNW, USA) from Loughborough University in UK (c), SAM from Empa in Switzerland (d).

\section{Measurement protocol}

All measurements conducted in this study were performed by the same experimenter traveling to each laboratory with the measuring equipment. This arrangement was necessary to prevent any measurement inconsistency due to differences in sensor characteristic, sensor application, and measurement protocol (Psikuta et al. 2014). Secondly, to minimise the impact of different chambers on the measurement outcome, air flow regime (horizontal piston and quasi-piston flow for Diana, Newton and SAM and diagonal mixing flow regime for Tore with inlet in the upper wall edge and outlet at the lower edge of the opposite wall) and the position of the manikin in the chamber were analysed for each individual case. The manikin location was used as recommended by the best practice of each laboratory. The set of environmental sensors (ThermCondSys5500, Sensor Electronic, Poland) was used in addition to laboratory own equipment to monitor ambient and radiant temperatures, relative humidity and air velocity in proximity of the manikin (about $60 \mathrm{~cm}$ in front and aside of the manikin) to confirm the required environmental conditions. The prerequisite for the ambient conditions in the chamber was that the radiant temperature deviates from ambient temperature by less than $1^{\circ} \mathrm{C}$ and the air velocity stays below $0.2 \mathrm{~m} / \mathrm{s}$ representing calm air conditions. Finally, four experiments were conducted using the nude manikin addressing the consistency of the heat flux measurement in manikins, lateral heat flow related to the heterogeneous surface temperature distribution, and manikin responsiveness during both passive and active manikin reactions. 


\section{Heat flux measurement in manikins}

A manikin intended for coupling with the physiological model must be able to provide a reliable measurement of the heat flux from its sectors as this parameter is the input to the model. A small discrepancy of up to $2 \%$ was demonstrated to have a negligible influence on the prediction accuracy of the skin, core temperatures as well as sweat rate (Psikuta et al. 2008). However, in that study the manikin (heated sweating cylinder) was a single sector device assumed to represent the entire human body (one value of heat loss for the entire body). In case of an anatomical device, such as a full body manikin, ideally, the detailed heat loss per sector should be used to fully benefit from a multi-sector device.

In this study, the heat flux from the nude surface of each manikin was measured using the instrumentation of each manikin at various environment-to-surface temperature gradients, such as $6-19^{\circ} \mathrm{C}$ for Tore, Newton and SAM, and $7-12^{\circ} \mathrm{C}$ for Diana to cover for small and large heat fluxes. The smaller range of tested temperature gradients for the manikin Diana resulted from the inability of this manikin to maintain a given surface temperature for gradients larger than $12^{\circ} \mathrm{C}$ due to a too small maximal heating power installed and using one resistance wire for measurement and heating. Each manikin was heated to a given constant and homogeneous surface temperature and the power input to maintain this temperature was used to calculate the resultant heat loss from the manikin surface. At the same time the ambient and radiant temperatures and air velocity were recorded $60 \mathrm{~cm} \pm 5 \mathrm{~cm}$ in front of the manikin.

\section{Heterogeneous surface temperature distribution}

To realistically simulate human thermal responses the manikin has to be able to operate with heterogeneous surface temperature distribution, i.e. with each sector set at an individual surface temperature. As most of the manikins were developed for measurements at constant and homogeneous surface temperature, the temperature-gradient driven heat exchange between sectors may become an issue. To evaluate this effect, the lateral heat flow between sectors was measured by comparing heat fluxes from respective sectors for measurements with homogenous and heterogeneous distribution of surface temperature.

The order of magnitude of the temperature gradients possible across various joints was simulated using the thermoregulation model (Fiala et al. 1999; Fiala et al. 2001; Psikuta et al. 2012). In the model, the virtual nude average person was exposed to chosen ambient conditions for an extended period of time of up to $3 \mathrm{~h}$ and subsequently the resultant temperature gradients across the neighbouring model compartments were determined. Since the prevailing phenomenon responsible for skin temperature gradients is vasoconstriction at extremities (Rintamaki 2007), the six joints at limbs, such as wrists, elbows, shoulders, ankles, knees and hips, were investigated.

Three tests at each ambient condition were performed that simulated the physiological surface temperature distribution during vasoconstriction (Figure 2). Each joint was investigated individually so that the possibly measured lateral heat flow could be attributed entirely to a particular joint. To investigate the lateral heat flow at a single joint the adjacent to this joint sectors of the manikin were heated to temperatures producing a given 
temperature difference (Figure 2). In addition, steady-state homogeneous measurements at the manikin surface temperature of $34^{\circ} \mathrm{C}, 33^{\circ} \mathrm{C}, 32^{\circ} \mathrm{C}$ and $30^{\circ} \mathrm{C}$ were carried out. These values were used as a reference for the heat loss of a particular sector when any lateral heat flow is prevented (all neighbouring sectors with the same surface temperature). Thus, any detected differences in heat loss between homogenous and heterogeneous surface temperature cases would suggest the presence of a lateral heat flow through a given joint. All measurements were performed at air temperatures of $25^{\circ} \mathrm{C}$ and $15^{\circ} \mathrm{C}$ and calm air conditions $(<0.2 \mathrm{~m} / \mathrm{s})$.

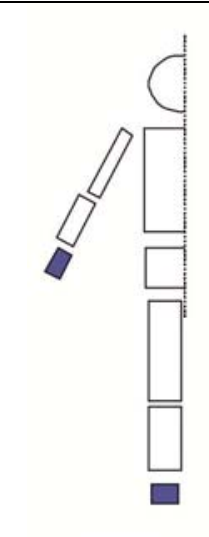

hands and feet at 25 and $15^{\circ} \mathrm{C}$

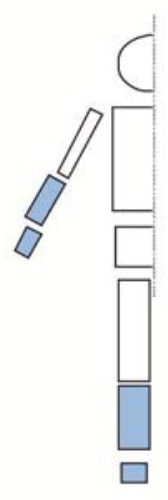

elbows

and knees

at 25 and $15^{\circ} \mathrm{C}$

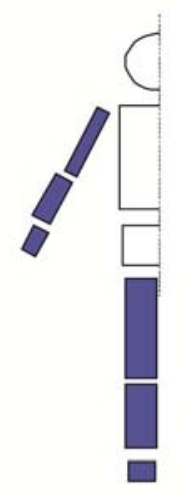

hips and

shoulders at $25^{\circ} \mathrm{C}$

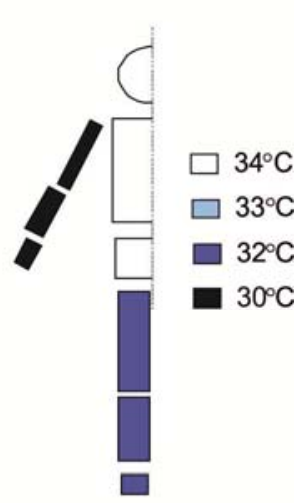

hips and

shoulders

at $15^{\circ} \mathrm{C}$

Figure 2. Scheme of heterogeneous surface temperature distribution in heterogeneous settings at air temperature of $25^{\circ} \mathrm{C}$ and $15^{\circ} \mathrm{C}$ and calm air conditions (below $0.2 \mathrm{~m} / \mathrm{s}$ ).

\section{Passive reaction}

This test was conducted to characterize the short-term response of the manikin skin temperature without active physiological control. Typically manikins are operated using a dedicated active control (PID) to maintain a given surface temperature of segments. Since none of the used manikins have been equipped with a cooling system, the decrease of the surface temperature can be executed only by controlled decrease of the heating or for faster surface temperature drop through switching off the heating. In the latter case, the manikin cools down passively, since there is no active system available to control the temperature course. A similar situation will occur when the surface temperature increase requires engaging the entire heating power available in the manikin heating system. In this case, the manikin can be heated with its maximal available rate without possibility of accelerating the surface temperature increase through active control. Besides, the course of the passive reaction depends on the thermal capacity of the manikin body including all materials used for its construction, such as metal skeleton, shells and all elements of heating, temperature measuring and sweating systems. Since the information on the amount of materials in manikin is difficult to quantify (e.g. the exact thickness/amount of material used for manikin shell is unknown, since they are manually made and may differ even between exemplars of the same type of manikin) and was not available for any of the manikins, the theoretical analysis of manikin’s thermal capacity was impossible. However, 
measuring the manikin passive thermal behaviour includes a resultant effect of all these factors and allowed a manikin comparison.

Both cases constitute limits of the manikin's active system and it is, therefore, important to evaluate if these issues impair or delay the surface temperature course in comparison to the thermal behaviour of human skin in various scenarios. Therefore, a series of simulations using the physiological model was conducted at extreme transient environmental conditions to determine the greatest temperature drop and increase rates at the human skin. Secondly, these rates were compared against the measured response times for the manikin operating beyond the limits of active control, i.e. when the heating system was switched off or operating at its maximal power output. This comparison allowed the determination of the application range of a given manikin when used with a physiological control. Two tests outside of the active control range were conducted, namely:

- Manikin cooled down to a surface temperature of $25^{\circ} \mathrm{C}$ in the climatic chamber at $25^{\circ} \mathrm{C}$, and was set to reach surface temperature of $34^{\circ} \mathrm{C}$. The rate of increase of surface temperature in the period corresponding to the manikin maximal power output was calculated.

- Manikin with surface temperature of $34^{\circ} \mathrm{C}$ was left to cool down in the climatic chamber at $25^{\circ} \mathrm{C}$ and still air conditions $(<0.2 \mathrm{~m} / \mathrm{s})$. The relative manikin surface temperature change was recorded.

A series of simulations under various activities (1-10 met) and environmental conditions (10-45 ${ }^{\circ} \mathrm{C}$ ambient temperature) were simulated to determine the maximal temperature changes possible to occur at the human skin at various body parts using the physiological model. The resultant manikin surface temperature change was compared to the physiological rates of change obtained using the model.

\section{Controlled reaction}

Since the majority of thermal manikins were developed for standardised measurements, most often conducted at steady-state conditions, their active control systems are tuned predominantly for precise maintenance of surface temperature and low adjustment rate of the heating power required to keep this temperature constant. When controlling such a manikin with a physiological model the surface temperature of a manikin is changed dynamically at a given time step rate, e.g. one minute. Depending on the manikin control system, the reaction of this system to frequent set-point changes may lead to either an over- or undershooting of the surface temperature or a substantial delay in reaching the new set-point temperature. To evaluate whether the manikin control system is properly tuned for operation with a physiological model, a series of step changes in surface set-point temperature was set and the resultant manikin surface temperature was measured. The chosen step changes in surface set-point temperature of $0.5,1$ and $2^{\circ} \mathrm{C}$ represented typical and extreme physiological skin temperature changes within one minute. The initial manikin surface temperature was $34^{\circ} \mathrm{C}$ and it was increased or decreased by the given step change in the climatic chamber at $25^{\circ} \mathrm{C}$. The time needed to reach the new given surface set-point temperature was reported. 
RESULTS

\section{Heat flux measurement in manikins}

Figure 3 shows the total and local heat flux released from the entire body, chest, thigh, and lower arm sectors at low ambient air velocity $(<0.2 \mathrm{~m} / \mathrm{s})$ and temperature gradients between $6-19^{\circ} \mathrm{C}\left(6-12^{\circ} \mathrm{C}\right.$ for Diana). The summary of total and local heat transfer coefficients measured for four tested manikins is listed in Table 2.
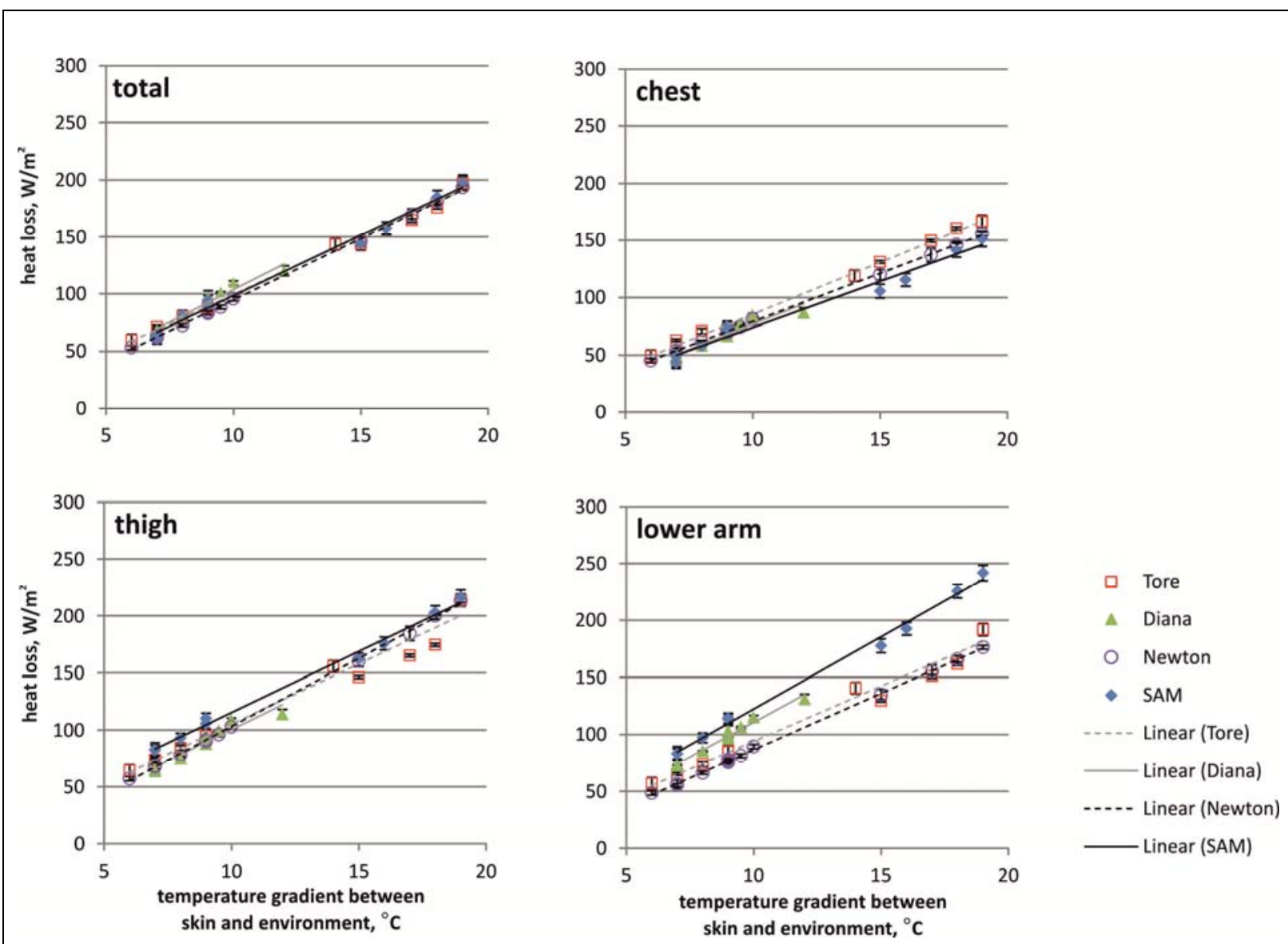

Figure 3. Total and local heat flux released from the entire body, chest, thigh, and lower arm sectors at low ambient air velocity $(<0.2 \mathrm{~m} / \mathrm{s})$ and temperature gradients between $6-19^{\circ} \mathrm{C}\left(6-12^{\circ} \mathrm{C}\right.$ for Diana). 
Table 2. Total and local heat transfer coefficient measured for four tested manikins and predicted by the physiological model at low ambient air velocity $(<0.2 \mathrm{~m} / \mathrm{s})$ and temperature gradients between $6-19^{\circ} \mathrm{C}\left(6-12^{\circ} \mathrm{C}\right.$ for Diana).

\begin{tabular}{|c|c|c|c|c|c|c|c|c|c|c|c|}
\hline$\left[\mathrm{W} / \mathrm{m}^{2} \mathrm{~K}\right]$ & head & chest & back & pelvis & $\begin{array}{l}\text { upper } \\
\text { arm }\end{array}$ & $\begin{array}{l}\text { lower } \\
\text { arm }\end{array}$ & hand & thigh & calf & foot & total \\
\hline model & 9.9 & 10.5 & 10.8 & 9.8 & 12.3 & 11.8 & 10.5 & 10.9 & 11.8 & 12.3 & 10.9 \\
\hline Tore & 5.5 & 9.1 & 10.3 & 11.5 & 9.4 & 9.7 & 11.6 & 10.6 & 6.3 & 9.3 & 10.2 \\
\hline Diana & 10.6 & 8.8 & 10.3 & 10.7 & 10.5 & 12.2 & 15.1 & 11.0 & 13.1 & 12.0 & 11.2 \\
\hline Newton & 8.6 & 8.4 & 9.5 & 9.6 & 12.3 & 9.9 & 15.3 & 12.0 & 12.4 & 11.4 & 10.7 \\
\hline SAM & 11.0 & 8.0 & 7.8 & 11.1 & 10.2 & 12.6 & $\mathrm{n} / \mathrm{a}$ & 10.7 & 12.1 & $\mathrm{n} / \mathrm{a}$ & 10.6 \\
\hline \multicolumn{12}{|l|}{ manikin } \\
\hline mean & 8.9 & 8.6 & 9.5 & 10.7 & 10.6 & 11.1 & 14.0 & 11.1 & 11.0 & 10.9 & 10.7 \\
\hline stdev & 2.5 & 0.5 & 1.2 & 0.8 & 1.2 & 1.5 & 2.1 & 0.8 & 3.2 & 1.4 & 0.4 \\
\hline
\end{tabular}

\section{Heterogeneous surface temperature distribution}

Figure 4 shows the differences in absolute heat loss from manikin sectors adjacent to the investigated joints between cases with homogenous and heterogeneous temperature distribution. For example, if the warmer sectors would heat up cooler sectors through lateral heat flow, the cool sectors should show heat gain (positive values in Figure 4) comparable to corresponding excessive heat loss at warm sectors (negative values in Figure 4). Since the hands and feet of the manikin SAM act as guards the evaluation of wrists and ankles was not performed. The maximal heating power of the manikin Diana was insufficient to maintain the required surface temperature between $30-34^{\circ} \mathrm{C}$ at ambient temperature of $15^{\circ} \mathrm{C}$, thus it was impossible to investigate effects of heterogeneous surface temperature distribution at higher magnitude of heat loss. 

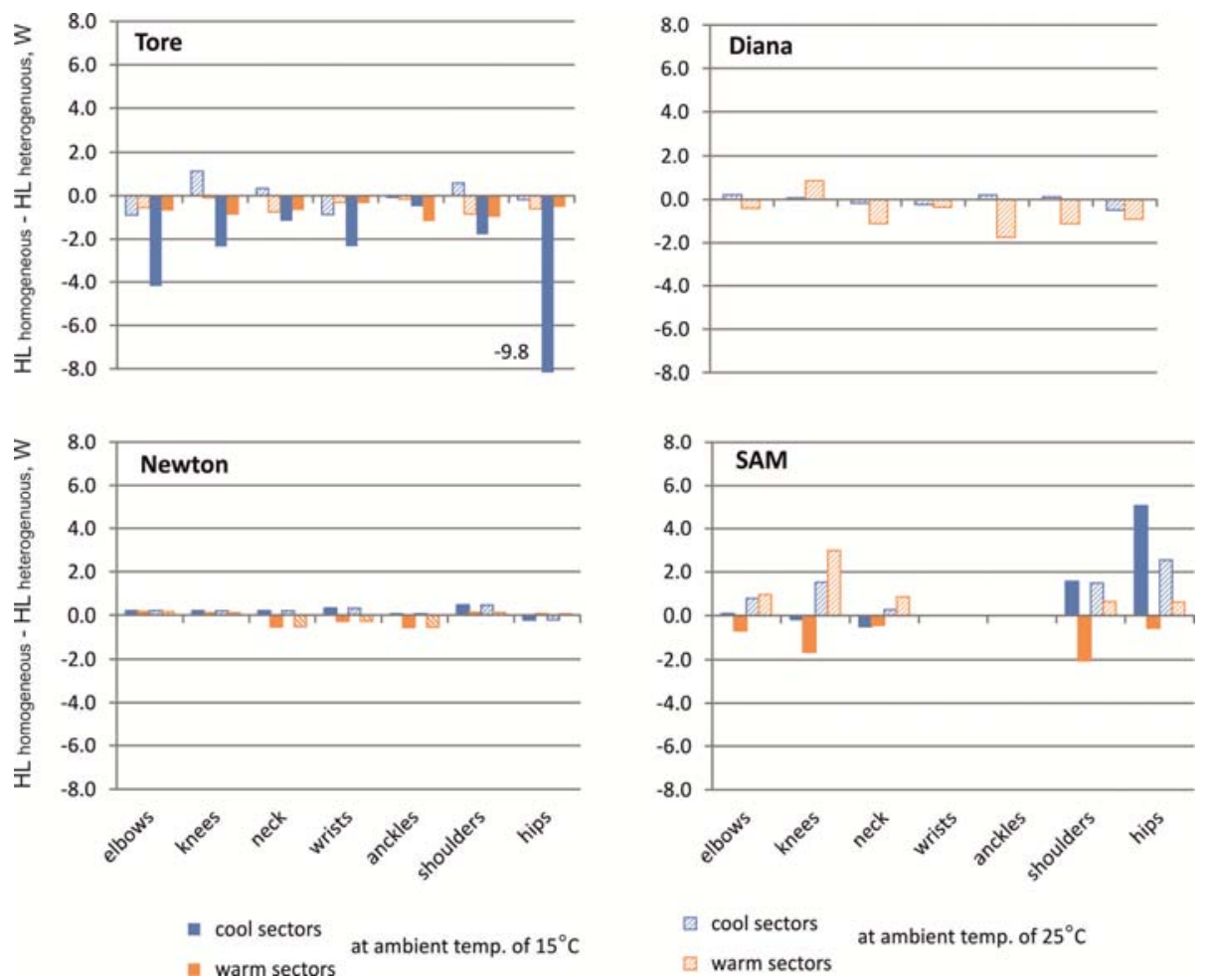

Figure 4. Differences in absolute heat loss [W] between homogeneous and heterogeneous temperature distribution cases from both warm and cool sectors adjacent to the investigated joints for four tested manikins at two ambient temperatures of 15 and $25^{\circ} \mathrm{C}$ and low ambient air velocity $(<0.2 \mathrm{~m} / \mathrm{s})$. Since hands and feet in manikin SAM act as guards the evaluation of wrists and ankles was not performed.

\section{Passive reaction}

Figure 5 shows the maximal temperature increment measured for the four evaluated manikins during heating up the manikin with maximal possible power output compared to the maximal temperature increment possible at the human skin for various body parts as given by the physiological model. Figure 6 illustrates an example of the course of the surface temperature drop measured in passively cooled manikins in the ambient environment at $25^{\circ} \mathrm{C}$ and low air movement $(<0.2 \mathrm{~m} / \mathrm{s})$ compared to the simulated thermo-physiological response of the human skin to the transient thermal conditions (a thermo-neutral nude person entering the environment at $25^{\circ} \mathrm{C}$ ). Table 3 lists the maximal possible deviation of the surface temperature of the manikin from that required by the physiological model at individual manikin sectors. This discrepancy emerges due to insufficient manikin passive cooling and is indicated in Figure 6 as a period of time during which the required cooling curve represented by the model is below the manikin actual cooling curve. 


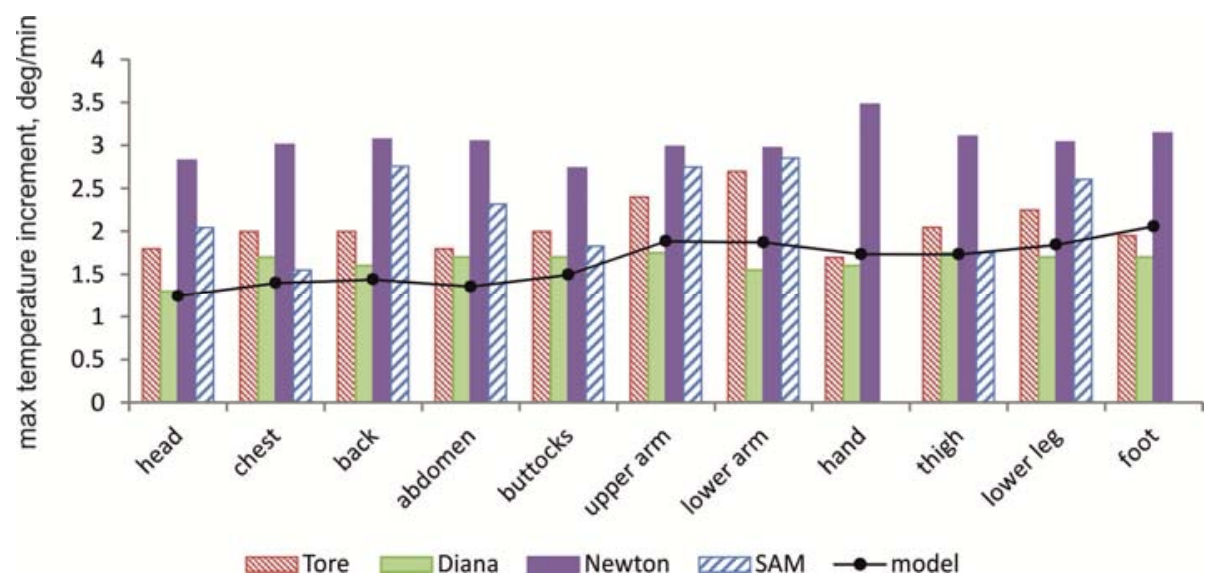

Figure 5. Maximal temperature increment measured for the four evaluated manikins during heating up the manikin with maximal possible power output compared to the maximal temperature increment possible at the human skin (model).

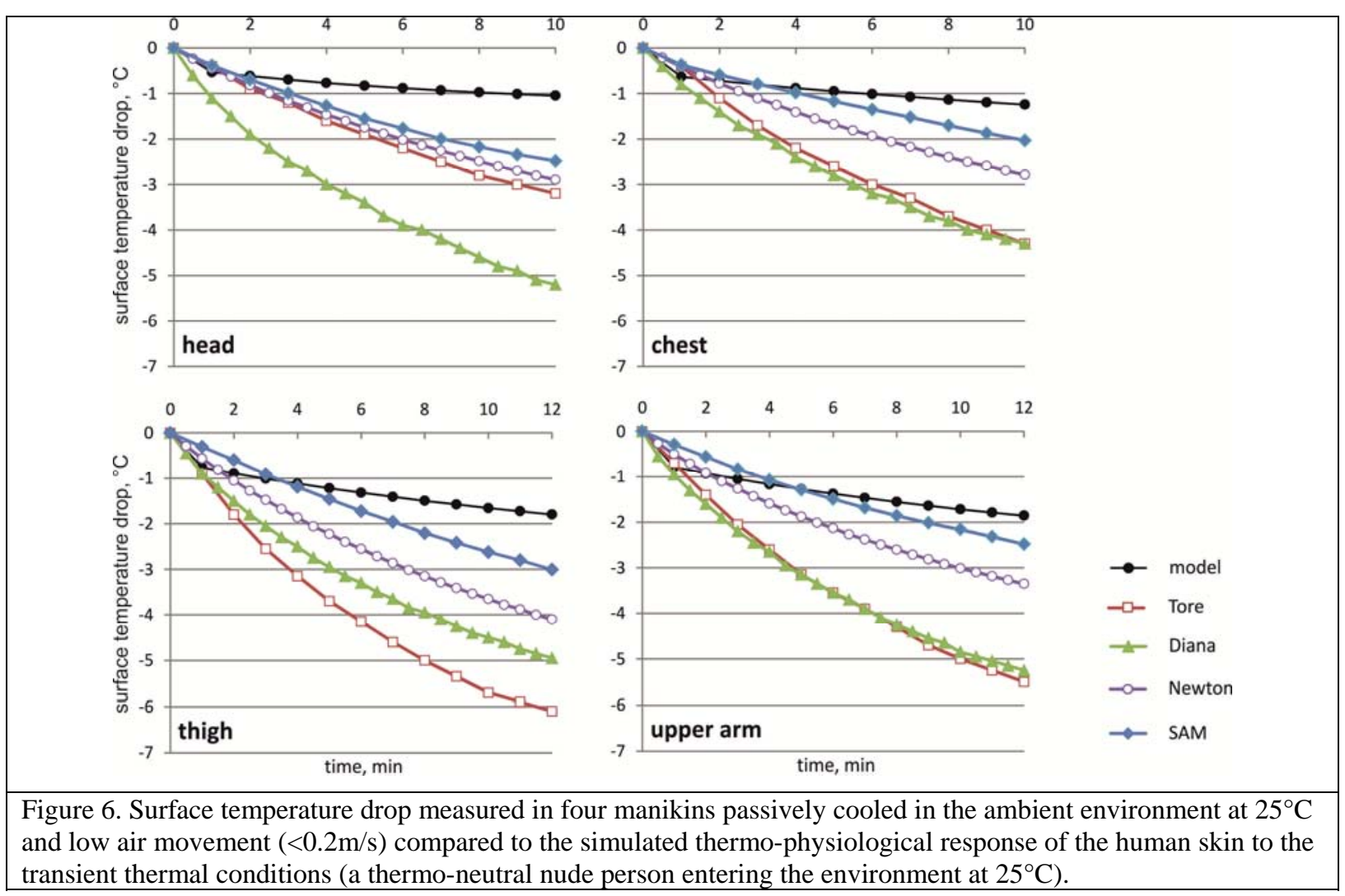


Table 3. Maximal discrepancy between the surface temperature reached by the manikin and required by the physiological model at individual manikin sectors due to insufficient manikin passive cooling (at ambient temperature of $25^{\circ} \mathrm{C}$ ).

\begin{tabular}{|c|c|c|c|c|c|c|c|c|c|c|c|}
\hline \multirow[b]{2}{*}[{}^{\circ}\mathrm{C}]{} & \multirow[b]{2}{*}{ head } & \multirow[b]{2}{*}{ chest } & \multirow[b]{2}{*}{ back } & \multirow[b]{2}{*}{ abdomen } & \multirow[b]{2}{*}{ buttocks } & \multirow{2}{*}{$\begin{array}{l}\text { upper } \\
\text { arm }\end{array}$} & \multirow{2}{*}{$\begin{array}{l}\text { lower } \\
\text { arm }\end{array}$} & \multirow[b]{2}{*}{ hand } & \multicolumn{3}{|c|}{ lower } \\
\hline & & & & & & & & & thigh & leg & foot \\
\hline Tore & -0.1 & -0.2 & 0.0 & 0.0 & 0.0 & -0.1 & 0.0 & -0.4 & 0.0 & 0.0 & -0.3 \\
\hline Diana & 0.0 & 0.0 & 0.0 & 0.0 & 0.0 & 0.0 & 0.0 & 0.0 & 0.0 & 0.0 & 0.0 \\
\hline Newton & -0.1 & -0.2 & -0.3 & -0.2 & -0.5 & -0.3 & -0.2 & 0.0 & -0.2 & -0.1 & -0.1 \\
\hline SAM & -0.1 & -0.4 & -1.1 & -0.9 & -0.6 & -1.2 & -0.6 & $\mathrm{n} / \mathrm{a}$ & -0.8 & -0.6 & $\mathrm{n} / \mathrm{a}$ \\
\hline
\end{tabular}

\section{Controlled reaction}

Figure 7 shows the time needed to reach the set-point temperature at manikin surface within a precision of $\pm 0.2^{\circ} \mathrm{C}$ for both positive and negative temperature step changes of $0.5,1$ and $2^{\circ} \mathrm{C}$ representing typical and extreme skin temperature variation within one minute. Due to the technical solution in the controlling of the manikin Tore, it was not possible to simulate step changes in set point temperatures.

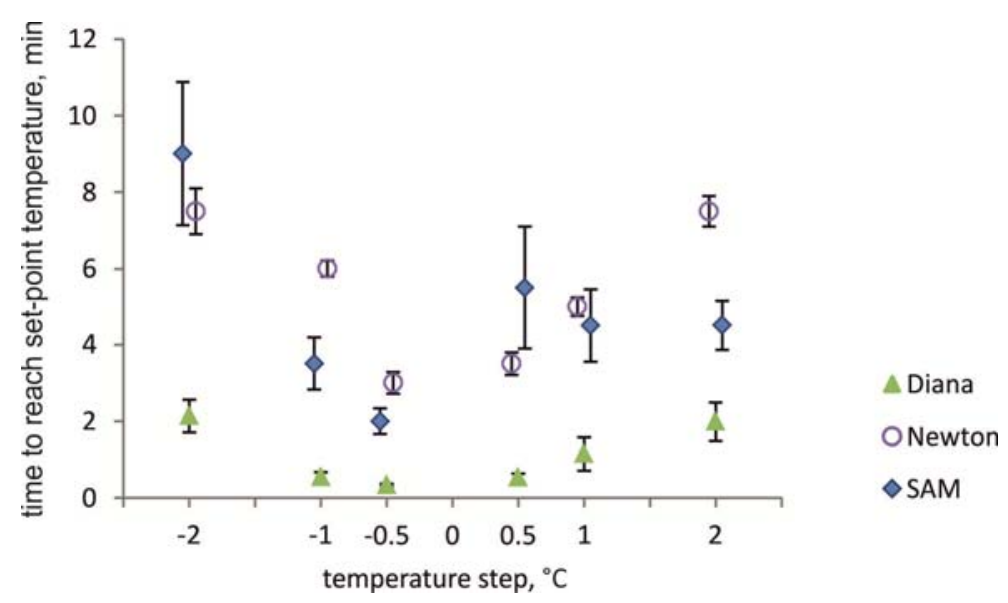

Figure 7. Time needed to reach the set-point temperature within $\pm 0.2^{\circ} \mathrm{C}$ precision for both positive and negative temperature step changes of $0.5,1$ and $2^{\circ} \mathrm{C}$ representing typical and extreme skin temperature variation. The error bars show the standard deviation of times for the manikin sectors. 


\section{DISCUSSION}

\section{Heat flux measurement in manikins}

All manikins measured highly repeatable and comparable total heat losses at temperature gradients between manikin surface and the environment in the range of $6-19^{\circ} \mathrm{C}$ for Tore, Newton and SAM and $6-12^{\circ} \mathrm{C}$ for Diana (Figure 3). The large individual sectors such as the trunk and thighs also show good agreement as opposed to smaller sectors at extremities, for example at lower arm, hand and foot (Figure 3 and Table 2). These discrepancies between manikins at the extremities can be related to several issues, such as, joint construction and its thermal guarding, which may have proportionally greater influence on smaller sectors. The joints of each manikin follow slightly different construction principles from continuous segments with or without flexibility (e.g. Diana’s elbows and hips, SAM’s shoulders), to rotating joints comprised of some openings for better flexibility (Tore's and Newton's shoulders, elbows and knees, Newton's hips), to discontinuously heated joints (Diana's knees and SAM's hips, knees and elbows, Tore's hips) (Figure 1). However, it was not possible to attribute the differences in heat transfer coefficient in neighbouring sectors to a particular joint type. Homogeneity of the environment in the manikin's climatic chamber, such as temperature stratification or local draughts, could also be a possible factor, which could not be detected by one spot measurement conducted in front of the manikin at the waist level in this study. On the other hand, all climatic chambers were developed with the special care for homogeneity of the ambient conditions as reported by the individual laboratories. To determine the actual reason for the variance in heat loss at extremities, more detailed technical information about production and construction process and possibly more measurements at several spots or entire area of the sectors would be required.

The maximal value for heat flux measurement inaccuracy that has negligible effect on prediction of the thermophysiological simulator was shown to be $2 \%$ (Psikuta 2009). To not exceed the level of variability observed in human trials (e.g. typical standard deviation in skin and core temperatures), a heat flux inaccuracy in the simulation using the manikin controlled by the physiological model should be lower than $5 \%$ (based on unpublished data of the first author). This value corresponds to the maximal heat loss increment that would result the mean skin or core temperature change by no more than a typical intra-subject variability of these temperatures in human trials.

Besides, in literature, different reference values for heat flux variation have been reported such as $4 \%$ of variation was proposed in international standards (ISO15831 2004), and 3-5\% for a good reproducibility in thermal insulation and moisture-vapour resistance (Anttonen et al. 2004; Fan and Qian 2004; Holmer and Nilsson 1995). The observed repeatability in measured heat flux for each manikin was between $2 \%$ and $5 \%$. Despite that the variance of total heat losses for all manikins was only $2 \%$, much larger variance of up to $91 \%$ was observed for the individual body parts with head, calves and hands having the greatest variance. The average variance of the largest sectors (trunk, upper arms and legs) accounting for $75 \%$ of the body area was $8 \%$. Therefore, given that all manikins would be coupled to an identical physiological model, they would all predict comparable physiological response with some exceptions for manikins or their sectors with markedly different heat transfer coefficients (Table 2). 
The most deviation was observed with manikin Tore: construction openings at the hips, elbows and knees might have caused some uncontrolled heat loss through inner ventilation when measured in the nude state. Possibly its difference to other manikins would diminish if tested with clothing preventing excessive ventilation of the manikin inner space. Secondly, the head showed the most variability between manikins. Since this body part is anatomically complex and was manufactured with various levels of details in the tested manikins, the wiring of surface temperature resistance sensor can be distinct (e.g. omitting or including the most pronounced parts such as nose, ears, and eyes). Besides, some extra material at fine face features can affect heat transfer through the heterogeneously thick manikin shell (e.g. Newton vs Diana) or additional features such as hair for manikin Tore may have increased head insulation and its surface roughness compared to the other manikins.

\section{Heterogeneous surface temperature distribution}

The comparison of heat loss from the manikin sectors under homogeneous and heterogeneous surface temperature conditions showed that there is a difference in heat loss between adjacent sectors upon the lateral temperature gradient. If our hypothesis that the warmer sectors heat up cooler sectors through lateral heat flow was true, the cool sectors should show heat gain (positive values in Figure 4) comparable to corresponding excessive heat loss at warm sectors (negative values in Figure 4). Nonetheless, the excessive heat losses and gains on warm and cool side of the joint, respectively, did not match for all manikins (Figure 4). This fact implies that the heat released at one body part flows not only to the adjacent body part but also in large part to the environment. This heat exchange was within $5 \%$ to $7 \%$ of the heat released at homogeneous surface temperature for manikin Newton, which is largely within the manikin heat flux accuracy of 5\% acceptable for physiological simulation. Thus, this manikin seems to be the most reliable amongst tested manikins followed by Diana (-26\% to 6\%), SAM (-15\% to 29\%) and Tore (-34\% to $7 \%$ ). The large heat exchange differences in SAM and Tore are plausibly related to their conductive metal joint construction and thermal guarding (large openings to the inside of Tore, joint guards regulated to the average temperature of neighbouring sectors in SAM). These technical issues should be solved for better performance of the manikins when controlled by the physiological model.

Tore's torso is hollow inside and the opening at the bottom is connected to an opening at the shoulders creating a possible “chimney effect”. The nude, warm body acting as a chimney in cool environment may have sucked in air from the hip area while there was a minimal effect around the exit at the shoulders as the internal air warmed up. Clearly, the effect was larger at $15^{\circ} \mathrm{C}$ (internal temperature of Tore was measured to be $29^{\circ} \mathrm{C}$ ), whereas at $25^{\circ} \mathrm{C}$ the effect was no longer observed (Figure 4). When the openings were closed while using a textile skin, internal temperature rose to $33-35^{\circ} \mathrm{C}$. In the case of Tore-type manikins there is a possibility to reduce internal ventilation by filling the hollow area with some fibrous insulating material to minimize air flow or to cover openings with a textile, e.g. Tyvek, taped to zones’ edges as in the ventilation study of Bouskill et al. (Bouskill et al. 2002). Around the elbows and knees there would not be the same effect as described above. In these areas the joints have wider gaps (knees) or unheated contact surfaces with no full coverage by the adjacent zone (elbow). Even in these cases the lower temperature gradient strongly diminished the effect suggesting that under clothing the difference would be 
even smaller. Thus, it is important to consider manikin's individual constructional characteristics and test conditions when testing and applying physiological model.

\section{Passive reaction}

All manikins showed sufficient heating power output to heat up body sectors at head and trunk at least as fast as required in simulated physiological scenarios (Figure 5). Since extremities react faster in their temperature increase, somewhat higher temperature increments were needed for these sectors. All tested manikins apart from Diana (upper and lower arms, hands, lower legs, and feet sectors) could fulfil this requirement. Despite the fact that the heating elements are very close to the manikin surface (heating and temperature measuring resistance wire in the outer coating), Diana showed insufficient heating capability, which was possibly related to its comparably low maximal power output $\left(200 \mathrm{~W} / \mathrm{m}^{2}\right.$, for other manikins $\left.350-800 \mathrm{~W} / \mathrm{m}^{2}\right)$ and interruptions in heating required for temperature measurement.

The surface temperature drop through passive cooling was sufficiently fast to follow the predicted skin temperature decrease at individual body parts (Figure 6). Some insufficient passive cooling occurred only at the initial period of exposure (up to 5 minutes); however, the difference between required and actual manikin surface temperatures was comparable to physiologically substantiated variance between human subjects, being typically $0.5-1^{\circ} \mathrm{C}$ for the mean and up to $2^{\circ} \mathrm{C}$ for the local skin temperatures (Figure 6 and Table 3). The passive cooling of the head and trunk was slightly faster than the cooling of extremities. This might be related to the fact that these segments directly face the environment, whereas the extremities partially face each other (legs) or other body parts (arms), thereby gaining heat through radiation.

\section{Controlled reaction}

Generally, the time needed to reach a set-point temperature was larger than the time required to mimick physiological skin temperature change. At ambient temperatures of $5-25^{\circ} \mathrm{C}$, the expected skin temperature change for various and even extreme scenarios (e.g. a strongly precooled person moving to a given environment and exercising to warm-up or an inactive thermo-neutral person exposed to a given environment to cool down) was approximately $0.5-2{ }^{\circ} \mathrm{C} / \mathrm{min}$. Only the manikin Diana fulfilled this requirement based on the results presented in Figure 7. This manikin was the fastest and most precise as indicated by the smallest spread of timing for individual body parts (see error bars in Figure 7). Manikins Newton and SAM had on average comparable times needed to reach set-point temperatures. However, Newton seemed to be more consistent within individual sectors. At the time of performing these measurements, manikin Tore had no option in its software to set various surface temperatures within one measurement, and hence, the transition between two set-point temperatures was not possible to measure. This test revealed the advantages and disadvantages of the different regulation strategies in tested manikins. The manikin Diana became less precise with the size of the step change, whereas Newton kept constant precision level over the tested step range. Both manikins showed a smooth change between steps without over- or undershooting. 
Manikin SAM, however, tended to over- and undershoot, which consequently, allowed it to reach the set-point temperature quicker than other manikins but it could not keep it precisely in the initial period of time. The results showed for SAM in Figure 7 include the time of the manikin overreaction until stabilisation at the new set-point temperature.

It should be noted that most parameters determined in these experiments are dependent on fixed properties of the manikin (e.g. heating/cooling speed) resulting from its construction. The dynamic regulation of surface temperature under extreme transient conditions, however, is determined largely by the settings of the PID controllers. The PID parameters, which currently are designed for optimal stability, can be adjusted through proper tuning to perform better in transients. Furthermore, PID performance is not crucial in the adaptive manikin operation principle if using heat flux as a set parameter and surface temperature as a feedback parameter at the cost of potentially inaccurate estimations of metabolic heat production over the body parts.

\section{CONCLUSIONS}

This manikin evaluation study revealed the opportunities and constraints of the tested thermal manikins with regards to their accuracy, precision and responsiveness. In general, the manikins evaluated in this study are suitable for coupling with a physiological model. The most important prerequisite for the coupling, which is a reliable heat flux measurement, was showed to comply with the required limits for acceptable accuracy of the physiological response with some exceptions of local heat transfer at distant extremities in Tore and at the head for all manikins. The lateral heat flow was not an issue for the manikin Newton, whereas all other manikins produced some increased heat exchange at joints. Nevertheless, when using clothing ensembles the environment-skin temperature gradients will diminish together with the heterogeneity of surface temperature, and hence, reduce the risk of uncontrolled heat loss. The manikin responsiveness evaluation revealed sufficiently fast response during passive heating and cooling for all manikins to simulate a change of human skin temperature. The dynamic regulation of surface temperature under extreme transient conditions was deficient in Newton and SAM. However, this parameter can be adjusted through proper PID tuning or is not crucial in the adaptive manikin operation principle if using heat flux as a set parameter and surface temperature as a feedback parameter.

As a recommendation, all thermal manikins intended for use as an adaptive manikin (human simulator) should undergo the presented evaluation procedure before being coupled with a physiological model and validated against human experimental data. The outcome of such an evaluation can be used to better understand the performance of the existing and future adaptive manikins and to trouble-shoot their potential problems. Finally, the proposed methodology is useful to improve the performance of the adaptive manikins and help to provide a reliable and versatile tool for the broad research and development in domains of clothing, automotive and building engineering. A possibility of evaluation of even very sophisticated garments and protective equipment in complex environmental scenarios by simply placing the manikin in the actual gear and environment is a major merit of this tool. Further, 24h operational readiness, high repeatability, low cost operation and high time effectiveness compared to human trials, and with no ethical concern seem to outweigh the investment cost. 


\section{ACKNOWLEDGMENTS}

The authors wish to thank Dr. Matthew Morrissey from Empa for fruitful discussions on improving the calibration protocol for the manikin SAM and consultation on scientific English writing.

\section{REFERENCES}

Anttonen $\mathrm{H}$ et al. (2004) Thermal manikin measurements--exact or not? International journal of occupational safety and ergonomics : JOSE 10:291-300

ASTMF1291-05 (2005) Standard Test Method for Measuring the Thermal Insulation of Clothing Using a Heated Manikin.

ASTMF2370-05 (2005) Standard Test Method for Measuring the Evaporative Resistance of Clothing Using a Sweating Manikin.

Blood K, Burke R (2010) Further validation of the model-controlled Newton thermal manikin against historical human studies. Paper presented at the 8th International Meeting for Manikins and Modelling, Victoria, Canada, 22-26 August 2010

Bouskill LM, Havenith G, Kuklane K, Parsons KC, Withey WR (2002) Relationship between clothing ventilation and thermal insulation Aihaj 63:262-268 doi:10.1080/15428110208984712

Burke R, Curran A, Hepokoski M (2009) Integrating an active physiological and comfort model to the newton sweating thermal manikin. Paper presented at the International Conference on Environmental Ergonomics, Boston, USA, 2-7 August 2009

Curran A, Peck S, Hepokoski M, Burke R Physiological model control of a sweating thermal manikin. Paper presented at the 10th Manikin and Modelling Meeting, Tampere, Finland, 7-9 September 2014

Fan JT, Qian XM (2004) New functions and applications of Walter, the sweating fabric manikin Eur J Appl Physiol 92:641-644 doi:10.1007/s00421-004-1134-1

Farrington R, Rugh J, Bharathan D, Burke R (2004) Use of a Thermal Manikin to Evaluate Human Thermoregulatory Responses in Transient, Non-Uniform, Thermal Environments Society of Automotive Engineers International 2004-01-2345

Fiala D, Havenith G, Broede P, Kampmann B, Jendritzky G (2012) UTCI-Fiala multi-node model of human heat transfer and temperature regulation Int J Biometeorol 56:429-441 doi:10.1007/s00484-011-0424-7

Fiala D, Lomas KJ, Stohrer M (1999) A computer model of human thermoregulation for a wide range of environmental conditions: the passive system Journal of Applied Physiology 87:1957-1972

Fiala D, Lomas KJ, Stohrer M (2001) Computer prediction of human thermoregulatory and temperature responses to a wide range of environmental conditions Int J Biometeorol 45:143-159 doi:10.1007/s004840100099

Foda E, Siren K (2012a) Design strategy for maximizing the energy-efficiency of a localized floor-heating system using a thermal manikin with human thermoregulatory control Energy Build 51:111-121 doi:10.1016/j.enbuild.2012.04.019

Foda E, Siren K (2012b) A thermal manikin with human thermoregulatory control: Implementation and validation Int J Biometeorol 56:959-971 doi:10.1007/s00484-011-0506-6

Gao C, Kuklane K, Wang F, Holmer I (2012) Personal cooling with phase change materials to improve thermal comfort from a heat wave perspective Indoor Air 22:523-530 doi:10.1111/j.1600-0668.2012.00778.x

Havenith G et al. (2013) Evaporative cooling: effective latent heat of evaporation in relation to evaporation distance from the skin Journal of Applied Physiology 114:778-785 doi:10.1152/japplphysiol.01271.2012

Havenith G et al. (2008) Apparent latent heat of evaporation from clothing: attenuation and "heat pipe" effects Journal of Applied Physiology 104:142-149 doi:10.1152/japplphysiol.00612.2007

Holmer I, Nilsson H (1995) Heated manikins as a tool for evaluating clothing Ann Occup Hyg 39:809-818 doi:10.1016/0003-4878(95)00041-0

ISO9920 (2007) Ergonomics of the thermal environment -- Estimation of thermal insulation and water vapour resistance of a clothing ensemble.

ISO15831 (2004) Clothing - Physiological effects - Measurement of thermal insulation by means of a thermal manikin. 
Keiser C, Becker C, Rossi RM (2008) Moisture transport and absorption in multilayer protective clothing fabrics Text Res J 78:604-613 doi:10.1177/0040517507081309

Konarska M, Soltynski K, Sudol-Szopinska I, Chojnacka A (2007) Comparative evaluation of clothing thermal insulation measured on a thermal manikin and on volunteers Fibres Text East Eur 15:73-79

Kuklane K, Heidmets S, Johansson T (2006) Improving thermal comfort in an orthopaedic aid: Better Boston brace for scoliosis patients. Thermal Manikins and Modelling.

McCullough EA The use of thermal manikins to evaluate clothing and environmental factors. In: 10th Conference on Environmental Ergonomics, Fukuoka, Japan, 2002. pp 427-430

McCullough EA, Jones B, Huck J (1985) A comprehensive database for estimating clothing insulation ASHRAE Transactions 91:29-47

Niedermann R, Psikuta A, Rossi RM (2014) Heat flux measurements for use in physiological and clothing research Int J Biometeorol 58:1069-1075 doi:10.1007/s00484-013-0697-0

Nilsson HO (2004) Comfort Climate Evaluation with Thermal Manikin Methods and Computer Simulation Models. University of Gävle and the Swedish National Institute for Working Life

Psikuta A (2009) Development of an 'artificial human' for clothing research. De Montfort University

Psikuta A et al. (2012) Validation of the Fiala multi-node thermophysiological model for UTCI application Int J Biometeorol 56:443-460 doi:10.1007/s00484-011-0450-5

Psikuta A, Niedermann R, Rossi RM (2014) Effect of ambient temperature and attachment method on surface temperature measurements Int J Biometeorol 58:877-885 doi:10.1007/s00484-013-0669-4

Psikuta A, Richards M, Fiala D (2008) Single-sector thermophysiological human simulator Physiological Measurement 29:181-192 doi:10.1088/0967-3334/29/2/002

Psikuta A, Wang L-C, Rossi RM (2013) Prediction of the Physiological Response of Humans Wearing Protective Clothing Using a Thermophysiological Human Simulator J Occup Environ Hyg 10:222-232 doi:10.1080/15459624.2013.766562

Redortier B, Voelcker T (2010) Implementation of thermo-physiological control on a multi-zone manikin. Paper presented at the 8th Manikin and Modelling Meeting, Victoria, BC, Canada, 22-26 August 2010

Richards MGM, McCullough EA (2005) Revised interlaboratory study of sweating thermal manikins including results from the sweating agile thermal manikin. Performance of Protective Clothing: Global Needs and Emerging Markets: 8th Symposium. doi:10.1520/stp12595s

Rintamaki H (2007) Human responses to cold Alaska medicine 49:29-31

Tanabe S, Arens EA, Bauman FS, Zang H, Madsen TL (1994) Evaluating Thermal Environments by Using a Thermal Manikin with Controlled Skin Surface Temperature ASHRAE Transactions 100:39-48 